\title{
Macrophage diversity in renal injury and repair
}

\author{
Sharon D. Ricardo, ${ }^{1}$ Harry van Goor, ${ }^{2}$ and Allison A. Eddy ${ }^{3}$
}

\begin{abstract}
${ }^{1}$ Monash Immunology and Stem Cell Laboratories (MISCL), Monash University, and Australian Stem Cell Centre, Clayton, Victoria, Australia. 2Department of Pathology and Medical Biology, University Medical Center Groningen, and University of Groningen, Groningen, The Netherlands. ${ }^{3}$ Division of Nephrology, Department of Pediatrics, University of Washington, and Seattle Children's Research Institute, Seattle, Washington, USA.
\end{abstract}

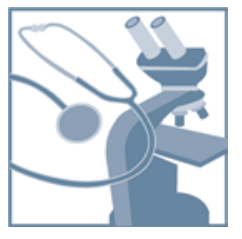

Monocyte-derived macrophages can determine the outcome of the immune response and whether this response contributes to tissue repair or mediates tissue destruction. In addition to their important role in immune-mediated renal disease and host defense, macrophages play a fundamental role in tissue remodeling during embryonic development, acquired kidney disease, and renal allograft responses. This review summarizes macrophage phenotype and function in the orchestration of kidney repair and replacement of specialized renal cells following injury. Recent advances in our understanding of macrophage heterogeneity in response to their microenvironment raise new and exciting therapeutic possibilities to attenuate or conceivably reverse progressive renal disease in the context of fibrosis. Furthermore, parallels with pathological processes in many other organs also exist.

Macrophages comprise a heterogeneous population of cells that belong to the mononuclear phagocyte system. They play an important role in tissue homeostasis and remodeling and are also potent immune regulators. Although widely recognized as contributing to the pathogenesis of renal fibrosis, glomerular and interstitial macrophages may also play beneficial, reparative, and matrix remodeling roles during tissue repair. There is compelling evidence that macrophages actively participate in the resolution of injury and promote tissue restoration in both immune- and non-immunemediated renal disease.

The heterogeneity of macrophages, their diverse roles in inflammation and tissue remodeling, and the coordinated activation and programming by other inflammatory cells is not fully understood. Functionally distinct subpopulations of macrophages, together with dendritic cells, may exist in the same tissue and play critical roles in both the initiation and recovery phases of scarring. The origin and activation state of the macrophage and the microenvironment in which they reside are critical determinants of their response to injury. Macrophages that secrete antiinflammatory cytokines, promote angiogenesis, and play a positive role in wound healing and tissue remodeling have been generally referred to as possessing an "alternative" phenotype. They are renowned for their heterogeneity and plasticity, which are reflected by their specialized functions in tissue inflammation and resolving injury. Macrophages have the ability to fuse with themselves and other cell types, particularly in response to inflammatory stimuli. Macrophages may therefore provide an important link between the bone marrow compartment and the regeneration of specialized cells of the kidney and other organs. This review discusses the heterogeneity of macrophages, their activation states, and diverse roles ranging from renal inflammation and replacement of damaged and apoptotic cells, to tissue remodeling. Fundamental insights into the therapeutic application of these antiinflammatory and reparative macrophage functions to renal diseases are discussed.

Nonstandard abbreviations used: AT1, angiotensin II receptor type 1; MCP-1, monocyte chemoattractant protein 1; SHIP, Src homology 2-containing inositol5'-phosphatase; Ym-1, chitinase 3-like 3.

Conflict of interest: The authors have declared that no conflict of interest exists. Citation for this article: J. Clin. Invest. 118:3522-3530 (2008). doi:10.1172/JCI36150.

\section{Macrophage origin and heterogeneity}

Macrophages are the oldest cell type in the hematopoietic system. Modern-day mammalian macrophages bear resemblance to the amebocytes in the circulation of horseshoe crabs (Limulus spp.) and have remained largely unchanged for millions of years (1). During early mammalian development, primitive macrophages appear to arise from a different cellular origin distinct from the blood monocyte (2-6). These primitive fetal macrophages have a high proliferative capacity and are derived from PU.1-negative hematopoietic cells (PU.1 is a tissue-specific transcription factor that is expressed in cells of the hematopoietic lineage) that lack monocytic cell surface markers (6-8). Apart from their role in the clearance of dying cells (9), fetal macrophages play a trophic role in promoting organ growth and nephrogenesis in the developing kidney (10). Once permanent or definitive hematopoiesis is established, the proliferative capacity of the macrophage declines and a distinct set of phagocytes, the monocyte-macrophages, are formed $(6,7,11)$.

Circulating monocytes derived from common bone marrow myeloid progenitors demonstrate a high cellular plasticity and can form tissue macrophages and dendritic cell subsets through a transdifferentiation process $(8,12,13)$. In addition, monocytes can differentiate into osteoclasts, which are fused polykaryons, as a result of an M-CSF- or RANKL-dependent cell-cell fusion process $(13,14)$. Monocytes themselves demonstrate antigenic and functional heterogeneity dependent on steady state or inflammatory cues. The recruitment of CCR $2{ }^{+} \mathrm{Ly}^{+}{ }^{+}$monocytes to sites of inflammation confirms that specific monocyte subsets are involved in an immune response or tissue remodeling (15). Sunderkotter et al. (16) reported that distinct subsets of monocytes distinguished by differential expression of Ly-6C may represent different stages of a continuous maturation pathway. Furthermore, a common monocyte progenitor characterized as $\mathrm{CX} 3 \mathrm{CR} 1^{+} \mathrm{CD} 117^{+} \mathrm{Lin}^{-}$has been described that can selectively differentiate into macrophage subsets and resident spleen dendritic cells (17).

During enhanced recruitment in response to disease states, inflammatory monocytes are recruited in response to cytokine cues and undergo differentiation into two broad but distinct subsets of macrophages that are categorized as either classically activated (M1) or alternatively activated (M2). M2 macrophages represent various phenotypes that are further subdivided into $\mathrm{M} 2 \mathrm{a}$ (upon exposure to IL-4 or IL-13), M2b (induced by immune com- 


\section{Table 1}

Macrophage activation states and functions

\begin{tabular}{|c|c|c|c|c|c|}
\hline $\begin{array}{l}\text { Macrophage } \\
\text { phenotype }\end{array}$ & Activation state & Stimuli & Phenotypic function & $\begin{array}{c}\text { Cytokine and } \\
\text { inflammatory profile }\end{array}$ & $\begin{array}{l}\text { Unique surface } \\
\text { markers }\end{array}$ \\
\hline M1 & Classical activation & $\begin{array}{l}\text { IFN- } \gamma+\text { LPS, } \\
\text { TNF, GM-CSF, } \\
\text { TLR/IL-1R ligand }\end{array}$ & $\begin{array}{l}\text { Proinflammatory } \\
\text { Th1 response }\end{array}$ & $\begin{array}{l}\text { IL-1, IL-12, IL-15, IL-18, IL-23, } \\
\text { TNF- } \alpha \text {, IL-6, MCP-1, CCL2, } \\
\text { CCL3, CCL4, CCL20/MIP-3a } \\
\text { ROS, NO, iNOS, NOS2 }\end{array}$ & $\begin{array}{c}\text { CD86, CD80, } \\
\text { MHC class IIIt, IL-1R, } \\
\text { IL-12 } \\
\text { ILi, IL-10 } 23^{\text {hi }}\end{array}$ \\
\hline \multirow[t]{3}{*}{ M2 } & $\begin{array}{l}\text { Alternate activation } \\
\text { (M2a polarization) }\end{array}$ & IL-4 or IL-13 & $\begin{array}{l}\text { Th2 responses, } \\
\text { type II inflammation }\end{array}$ & $\begin{array}{c}\text { Fibronectin, BIG-H3, } \\
\text { arginase-1, TNF- } \alpha, \text { IL-6, } \\
\text { IGF, CCL13/MCP-4, CCL22, } \\
\text { CCL18, } \beta 2 \text { integrins }\end{array}$ & $\begin{array}{c}\text { Mannose receptor, } \\
\text { scavenger receptor, MHC } \\
\text { class II }{ }^{\text {hi }} \text {, decoy IL-1R11, } \\
\text { FIZZI/Ym-1 }\end{array}$ \\
\hline & $\begin{array}{l}\text { Type II activation } \\
\text { (M2b polarization) }\end{array}$ & $\begin{array}{l}\text { Immune complex } \\
+ \text { TLR/IL-1R ligands }\end{array}$ & $\begin{array}{l}\text { Immunoregulation, } \\
\text { Th2 activation }\end{array}$ & $\begin{array}{l}\text { IL-10, TNF- } \alpha, \text { IL-1, IL-6, } \\
\text { IL-12, SPHK1, CCL1 }\end{array}$ & $\begin{array}{l}\text { CD86, MHC class II hi, } \\
\text { IL-10 }\end{array}$ \\
\hline & $\begin{array}{c}\text { Deactivated } \\
\text { (M2c polarization) }\end{array}$ & $\begin{array}{l}\text { IL-10, TGF- } \beta \text {, } \\
\text { glucocorticoids }\end{array}$ & $\begin{array}{l}\text { Immunosuppression, } \\
\text { matrix remodeling, } \\
\text { tissue repair }\end{array}$ & $\begin{array}{l}\text { IL-10, IL-1 } \beta, \text { IL-6, TGF- } \beta \text {, } \\
\text { ECM proteins, CCL16, } \\
\text { CCL18, arginase-1 }\end{array}$ & $\begin{array}{l}\text { SLAM (CD150), mannose } \\
\text { receptor, MHC class IIlo }\end{array}$ \\
\hline
\end{tabular}

BIG-H3, fasciclin domain 4 protein; MIP, macrophage inflammatory protein; SLAM, signaling lymphocytic activation molecule; SPHK1, sphingosine kinase 1. Note that $F I Z Z I$ and $Y m 1$ gene expression is characteristic of the alternative pathway of macrophage activation.

plexes in combination with IL-1 $\beta$ or LPS), and M2c cells (following exposure to IL-10, TGF- $\beta$, or glucocorticoids) $(18,19)$, as detailed in Table 1 . The plasticity and differentiation of macrophages into M1 and M2 functional phenotypes therefore represent extremes of a continual spectrum of differential pathways.

Activation of M1 macrophages by classical immune pathways involves an IFN- $\gamma$-dependent Th1-type response. Exposure to IFN- $\gamma$ and LPS or cytokines TNF and GM-CSF induces M1 polarization that is characterized by the production of IL-12 and IL-23, both known to be produced by APCs. The capacity of macrophages and dendritic cells to produce IL-12 and IL-23 strongly influences the outcome of the Th1, Th17, and $\mathrm{CD}^{+} \mathrm{T}$ cell response. In addition to Th1 cells, IL-23 and Th17 cells play an important role in the pathogenesis of autoimmune disorders and renal allograft (20). In addition, neutralizing anti-IL-12/23p40 antibodies have been used successfully to treat psoriatic skin inflammation $(21,22)$. This is a fundamental response paradigm in cellular immunity and in delayed-type hypersensitivity responses that cause tissue damage. For example, in the kidney these processes mediate crescentic glomerulonephritis and acute allograft rejection. In the classical M1 pathway, activation by IFN- $\gamma$ is crucial; together with a microbial trigger, it induces expression of MHC class II antigens and proinflammatory cytokines.

The alternative M2 macrophage activation pathway typically deactivates macrophages after exposure to Th2-type cytokines. Such responses characterize immunoregulatory, immunosuppressive, and protumoral settings (19). M2 cells induced by exposure to IL-4 and IL-13 (M2a) and deactivating cytokines such as IL-10 and TGF- $\beta$ (M2c) are thought to suppress immune responses and promote tissue remodeling $(19,23-26)$. A role for the NF-KB activator IKK $\beta$ has also been implicated in the promotion of an alternative immunosuppressive phenotype (27). Macrophage reactivation during antiinflammatory responses may occur as a consequence of innate or acquired immune responses, often characterized by macrophage uptake of apoptotic cells or lysosomal storage of host molecules. Unlike its classically activated counterpart, the M2 macrophage may help to resolve inflammation through high endocytic clearance capacities and production of trophic factors, together with reduced proinflammatory cytokine secretion (28). For example, M2 macrophages may secrete trophic factors that promote angiogenesis and mediate wound healing by promoting ECM remodeling (29). M2 macrophages also express fibronectin 1 $(\mathrm{FN}-1)$, the TGF- $\beta$-induced matrix-associated protein BIG-H3, and IGF-1, which provide signals for tissue repair and proliferation. (25). They can generate arginase-1, which suppresses inflammation by inhibiting the production of proinflammatory $\mathrm{NO}$ (29). Furthermore, M2 cells express the IL-1 receptor antagonist, which inhibits the effects of the proinflammatory cytokine IL-1, the mannose receptor, and chitinase 3 -like 3 (Ym-1) (30). It has recently been reported that a subset of adipose tissue macrophages exhibiting an M2 phenotype produce MMP-9, which in the kidney may contribute to attenuation of fibrotic lesions (31).

As a key component of the inflammatory response that determines tissue destruction or recovery, increasing evidence suggests that macrophages do not remain committed to a single activation state. They may regress to a resting state that can subsequently be reactivated another way. Following phagocytosis of apoptotic cells, classically activated M1 macrophages may revert to an M2 activated state (32). Tumor-associated macrophages (TAMs) also provide evidence of a bidirectional transformation between antiinflammatory and immunosuppressive phenotypes $(33,34)$. Stemming from a common myeloid precursor, there are distinct subpopulations of monocyte-derived TAMs with different functional phenotypes dependent on the site of tumor origin and progression of disease. TAMs share a phenotype similar to that of M2 macrophages and are likely to represent a unique myeloid cell differentiation program (35). Tumor-conditioned granulocytes may play a role in priming macrophages toward either an M1 or M2 phenotype (36).

\section{Reparative role of macrophages in tissue remodeling}

Inflammatory cues within the regional microenvironment can prime macrophage phenotype and determine whether these cells will have a beneficial or deleterious effect during tissue repair and remodeling. Macrophage phenotype and function ultimately determine the outcome of inflammation and the development of irreversible tissue scarring (Figure 1). Unselective macrophage depletion, via the admin- 


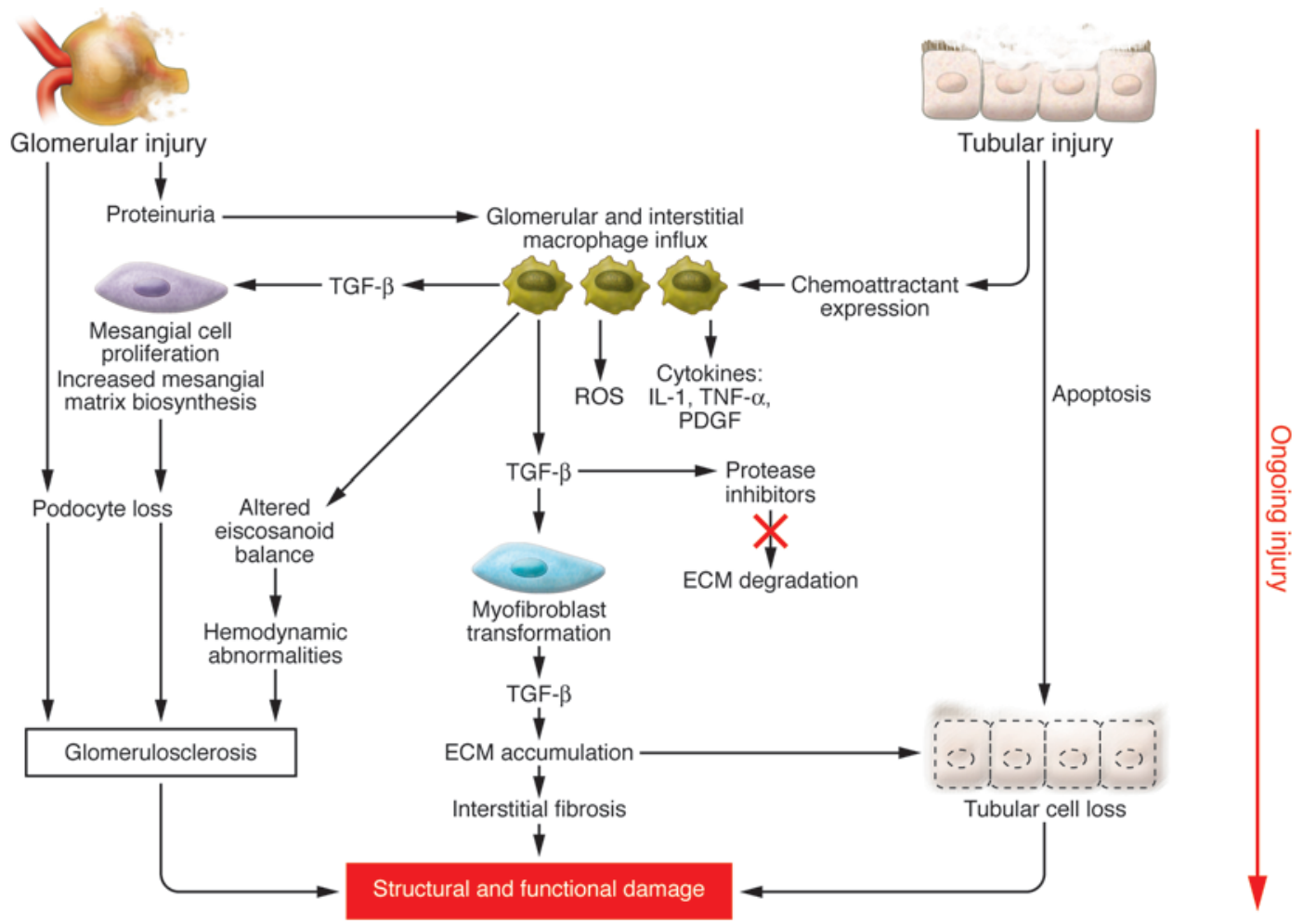

Figure 1

The relationships between infiltrating macrophages and macrophage-derived products in chronic ongoing inflammation lead to structural and functional renal damage. In response to tubular and glomerular injury/dysfunction, macrophage chemoattractants and proteinuria promote the infiltration of renal macrophages, leading to the generation of proinflammatory cytokines, vasoactive eicosanoids, and ROS. The initial injury and proinflammatory state may lead to podocyte and tubular cell apoptosis. Overproduction of TGF- $\beta$ by macrophages, myofibroblasts, and mesangial cells promotes increased synthesis of glomerular and interstitial ECM proteins and decreased matrix turnover due to the synthesis of matrix-degrading protease inhibitors. The net effect of interstitial fibrosis and/or glomerulosclerosis and podocyte and tubular cell loss is the disruption to tissue architecture and loss of renal function.

istration of anti-macrophage serum or liposomal clodronate, can reduce experimental acute kidney damage by abrogating persistent inflammation and the subsequent development of fibrosis (37-39). Complete macrophage depletion by sublethal irradiation prevents the influx of macrophages to an injured kidney and decreases fibrosis severity $(40,41)$. However, a selective approach to macrophage depletion will provide greater insight into the role of functionally distinct subpopulations of macrophages that contribute to injury inducing and tissue remodeling phases of inflammatory scarring. Conditional macrophage depletion based on transgenic expression of diphtheria toxin highlights the importance of scar-associated macrophages for recovery responses such as matrix degradation following liver injury (42). Hepatic macrophages were shown to be essential for matrix regression during the recovery phase of experimental hepatic fibrosis (42) and to regulate stellate cell proliferation (43). Within muscle, macrophages enhance myogenic growth by releasing trophic factors that stimulate myogenic precursor cells (44). Using in vivo tracing methods, Arnold et al. (45) showed that CX3CR1 ${ }^{\text {hi Ly- } 6 C^{+}}$inflammatory macrophages initially recruited into skeletal muscle are able to rapidly switch to an antiinflammatory $\mathrm{M} 2$ phenotype in response to their changing microenvironment.

Macrophages and their trophic factors are implicated in injury resolution and cellular restoration in a number of organs (46-49). During Schistosoma spp. infection, M2 macrophages attenuate organ injury by downregulating inflammation, predominantly the Th1 response (50). The effect of $\mathrm{M} 2$ skewing has been investigated using Src homology 2-containing inositol-5' -phosphatase-null (SHIP-null) mice (51). SHIP is essential for endotoxin tolerance; it dampens LPS-induced M1 activation of bone marrow-derived macrophages. Macrophages from SHIP-null mice manifest an M2 phenotype with constitutively high arginase I and Ym-1 levels and require a TGF- $\beta$-rich environment during differentiation.

\section{Opposing roles of macrophages in kidney disease and repair}

Macrophages promote renal fibrosis, and most interstitial and glomerular renal diseases are characterized by macrophage accumulation. Traditionally, these macrophages are considered transients that enter glomerular or interstitial areas to modulate immune responses and/or process debris and apoptotic cells generated as a result of the primary kidney insult. However, in the face of ongoing injury, sustained macrophage infiltration may result in the continuous production of various wound-healing growth factors. Ultimately this initial process of wound healing becomes pathological, resulting in irreversible fibrosis, tissue destruction, and progressive chronic kidney disease (Figure 1). What begins as an initially essential and beneficial influx of macrophages transforms into their extended presence with damaging consequences. 
The progression of immune-mediated renal disease involves an interplay between infiltrating $\mathrm{T}$ cells, dendritic cells, and macrophages, which contributes to the immunopathogenesis of glomerulonephritis (52-54). T cell activation is dependent on dendritic cells in secondary lymphoid organs and resident dendritic cells in the kidney $(8,52,55)$. Renal allograft rejection is a T cell-dependent process resulting in graft injury through cytotoxic mechanisms and by $\mathrm{T}$ cell activation and macrophage effector function $(56,57)$. Tipping and Holdsworth (58) identified a crucial role for $\mathrm{CD}^{+} \mathrm{Th} 1$ cells and macrophages in a delayed-type hypersensitivity mechanism of crescent formation in experimental glomerulonephritis. T cell responses can initiate hypersensitivity immune reactions and stimulate macrophages to generate proinflammatory mediators of injury. During the ensuing damage, macrophages are also the predominant cell type responsible for the development of fibrosis and progressive fibrotic scarring.

Members of the TGF- $\beta$ superfamily are the most extensively studied macrophage-derived growth factors that have been linked to renal fibrosis (59). Macrophages, tubular epithelial cells, and myofibroblasts are all capable of synthesizing TGF- $\beta$ at different stages during the development of renal fibrotic lesions (60). However, the observation that macrophage ablation markedly attenuates fibrosis in various conditions suggests that these cells are among the main producers of this growth factor $(40,41)$. Once activated, TGF- $\beta$ signals through transmembrane receptors that activate the Smad proteins that regulate the transcription of important target genes including those that encode collagens. In the kidney, macrophage-derived TGF- $\beta$ may promote fibrosis by paracrine activation of matrix-producing myofibroblasts and promotion of tubular epithelial cell transdifferentiation into myofibroblasts $(61,62)$. The downstream accumulation of TGF- $\beta$-induced ECM is generally considered destructive in nature (63). However, macrophages may synthesize and secrete collagens themselves (64). TGF- $\beta$ can also deactivate macrophages (65) and induce a tissue-stabilizing, antiinflammatory macrophage phenotype characterized by production of collagen type VI (66).

Modulating macrophage phenotype and function has been reported to reduce renal injury in models of renal disease, including glomerulonephritis $(67,68)$, allograft injury (69), and interstitial fibrosis (70). In a model of obstructive nephropathy, Nishida et al. (71) showed that angiotensin II receptor type 1-expressing (AT1-expressing) macrophages had a protective effect in later stages of fibrotic injury. Transplantation of bone marrow from AT1-null mice into wild-type mice led to more severe interstitial fibrosis despite reduced numbers of monocytes and macrophage progenitors, compared with mice reconstituted with AT1-positive wild-type bone marrow (71). Similar detrimental effects have been reported when obstructive nephropathy was induced in mice lacking the classical urokinase receptor (uPAR) compared with wild-type mice (72).

Wang et al. (73) provided direct evidence that ex vivo manipulation of macrophages can reduce renal injury and facilitate repair by using adoptive transfer studies of M2-polarized macrophages injected into mice with chronic inflammatory renal disease. Splenic macrophages stimulated with IL-4/IL-13 were injected systemically after the onset of disease, where they were found to downregulate inflammatory cytokine and chemokine expression of the host infiltrating macrophages (73). Therefore, the protective effect of the transplanted macrophages was associated with M2-skewing of host macrophages, supporting findings in models of glomerular disease $(74,75)$. In a rat model of nephrotoxic nephritis, the transfer of macrophages transduced with an NF- $\mathrm{KB}$ inhibitor resulted in a reduction in iNOS and MHC class II expression in glomeruli (74). Although the injected antiinflammatory macrophages comprised only $15 \%$ of the glomerular macrophages, they significantly reduced glomerular infiltration and activation of host macrophages, resulting in the attenuation of renal injury (74).

\section{Bone marrow-derived cells in kidney repair}

The kidney has a remarkable ability to regenerate following acute injury. Most notably, the renal epithelia have the intrinsic capacity to rapidly self-duplicate (76). Renal injury and repair comprise a delicate balance between cell loss and proliferation and macrophage-dependent interstitial matrix accumulation and remodeling. Whether bone marrow-derived cells and/or infiltrating macrophages can contribute directly to the replacement of injured and dying tubular epithelia and glomerular cells by transdifferentiation and/or cell-cell fusion is an active area of investigation (Figure 2).

Bone marrow-derived cells are thought to cross lineage boundaries and transdifferentiate, resulting in a phenotype switch in response to inflammatory cues to repair injured organs, including the kidney (77-80). Cell-tracing studies using either Y chromosome tracking in sex-mismatched human kidney transplants $(81-83)$ or $\mathrm{GFP}^{+}$reporter mice $(72,84,85)$ provide evidence that bone marrow-derived cells can replace the renal vasculature and interstitial cells $(81,84,85)$, renal tubular epithelial cells $(77,82$, 83 ), and cells of the glomerulus (77). Masuya et al. (86) showed that a single hematopoietic cell was capable of differentiating into mesangial cells in lethally irradiated recipient mice. In all studies, hematopoietic engraftment of the host is needed before bone marrow cell engraftment into the kidneys occurs, suggesting that it is the hematopoietic progeny of bone marrow-derived cells and not the stem cells themselves that engraft into host tissues (87). There is evidence that bone marrow-derived cells normally reconstitute mesangial and interstitial cells $(88,89)$. Imasawa et al. (88) demonstrated that $\mathrm{GFP}^{+}$bone marrow cells migrate to glomeruli and interstitium and contribute to the normal cell turnover.

Although the majority of regenerating tubular epithelial cells are derived from an intrarenal source $(90,91)$, bone marrowderived cells may contribute to the replacement of tubular epithelial cells through a process of cell fusion $(92,93)$. The hematopoietic cell type that is responsible for the cell fusion-derived epithelial cells is unclear, although growing evidence suggests that macrophages are involved, as has been shown in the liver (93, 94). Recently, Li et al. (92) used cre/loxP recombination under the direction of the kidney-specific cadherin promoter with sexmismatched bone marrow transplantation to demonstrate that fusion occurs in post-ischemic kidneys and in cocultures of bone marrow cells and renal epithelial cells in vitro. Macrophages demonstrate cell plasticity and have the ability to undergo cellcell fusion with themselves or other cell types, particularly in response to inflammatory stimuli (95). Mature blood monocytes and inflammatory macrophages have been shown to transform into vascular elements including endothelial cells, myofibroblasts, and smooth muscle cells in addition to neuronal and liver cells $(87,96-98)$. Taken together, the discovery that cell fusion events occur between renal cells and macrophages or their highly proliferative progenitors suggests a scientific basis for new cell therapy approaches for organ regeneration. 


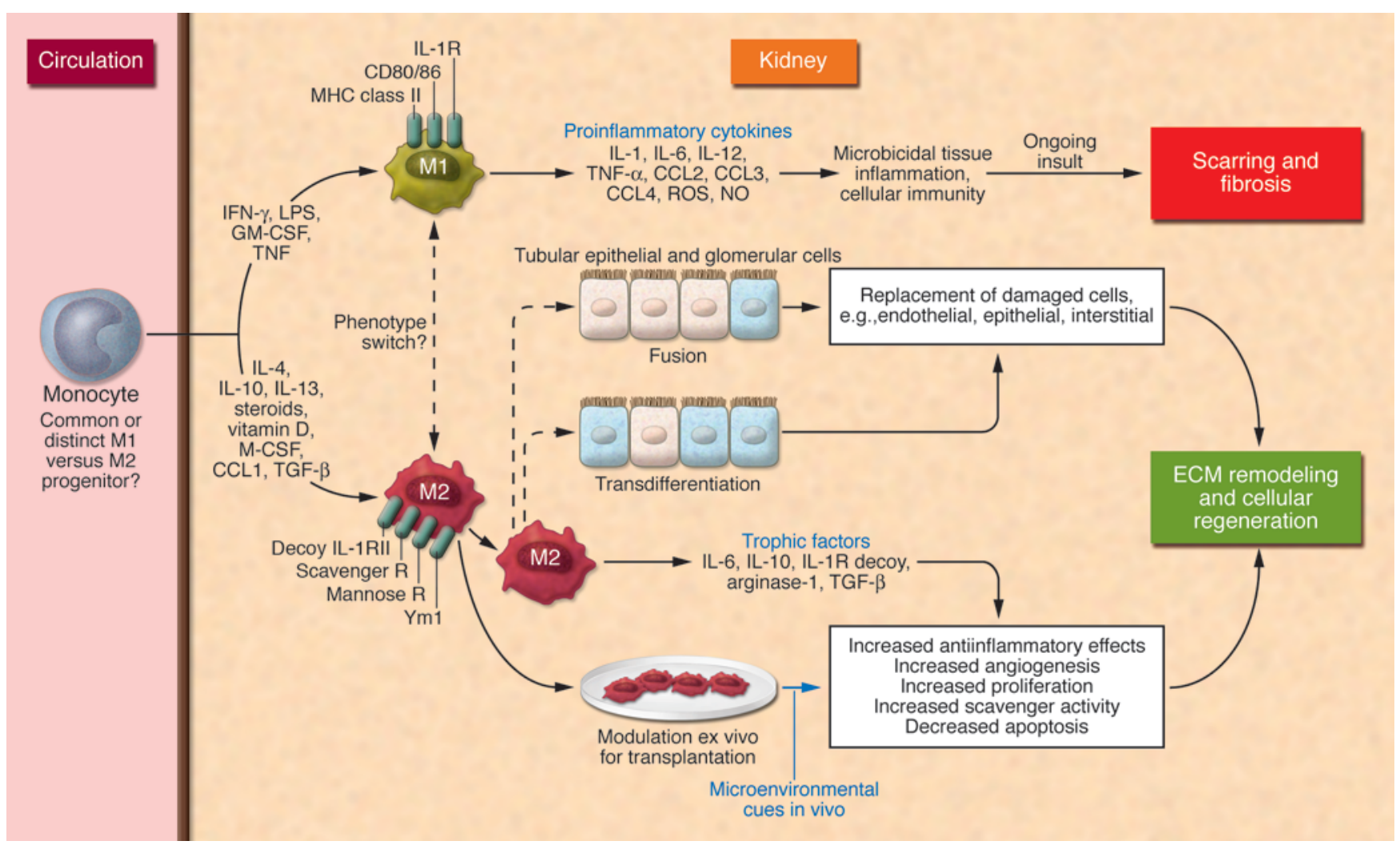

Figure 2

Macrophage phenotype and function are critical determinants of fibrotic scarring or resolution of injury. Monocytes from the circulation that enter the kidney in response to inflammatory cues undergo distinctive pathways of differentiation into classically activated M1 macrophages or the alternative M2 phenotype. Activation of M1 inflammatory macrophages by classical immune pathways may lead to the expression of MHC class II antigens and release of proinflammatory cytokines. In response to ongoing injury, M1 macrophages propagate inflammation and ultimately the development of fibrosis. Dependent on microenvironmental cues, M2 macrophages may be recruited from the circulation or activated in situ as a result of an M1-to-M2 phenotype switch. M2 antiinflammatory macrophages secrete regenerative trophic factors that promote cell proliferation and reduce apoptosis and stimulate angiogenesis. Macrophages derived from engrafting bone marrow myeloid progenitors may contribute to the repopulation of injured tubular epithelial and glomerular cells by a process of transdifferentiation or cell-cell fusion, leading to replacement of damaged cells. Ex vivo modulation of macrophages to form an M2 phenotype for transplantation may be used therapeutically to suppress the immune response and promote tissue remodeling, leading to structural repair and functional recovery. $\mathrm{R}$, receptor.

\section{In search of the beneficial role of macrophages: implications for renal therapy}

Recognition of macrophage functional diversity offers the possibility of new therapies for patients with chronic kidney disease given the nearly universal colocalization of interstitial macrophages within regions of kidney fibrosis and nephron destruction (60). However, it must be acknowledged that the M1/M2 macrophage phenotype and function paradigm is based largely on studies in mice; extrapolation to man must proceed with caution (99). An additional challenge pertains to the fact that although an M2-skewed macrophage response is associated with inflammation resolution and tissue healing, these cells also promote fibrosis. The mechanisms that fine-tune the M2-associated response to achieve repair without scarring and long-term consequences are unknown and demand attention. Assuming that these questions can be answered, it is easy to envisage novel cellular therapies based on the infusion of preprogrammed macrophages or molecular therapies that program or mimic the macrophage phenotype in vivo. The goal of discovery of effective treatment for progressive disorders such as chronic kidney disease should guide future investigations in several areas.

\section{Engineering monocytogenesis}

It is assumed that the majority of kidney injury-associated macrophages are derived from the circulating monocyte pool, as resident kidney interstitial macrophages and dendritic cells are usually considered terminally differentiated and nonproliferating. Derived from $\mathrm{CD} 34^{+}$bone marrow progenitors, it is unclear whether the M1 and M2 macrophages originate from common or distinct lineages. In either case, interventions designed to block M1 generation or enhance M2 polarity may be therapeutically feasible (Figure 3).

One can envisage cellular therapies based on the infusion of peripheral monocytes primed ex vivo by exposure to a cocktail of cytokines (IL-4 and IL-13, for example) to induce M2 macrophages, as reported in a mouse model of kidney disease (73). PPAR $\gamma$ exposure has been reported to skew monocytes toward an antiinflammatory phenotype (100). Ideally, such cells would be enriched for specific chemokine/chemoattractant receptors that facilitate the preferential migration of these cells to sites of kidney damage. Such an approach could be aided by the identification of new M2 phenotypic markers that also confer biological functions associated with tissue repair. As previously mentioned, the recent 
A Cell-based therapy following kidney injury

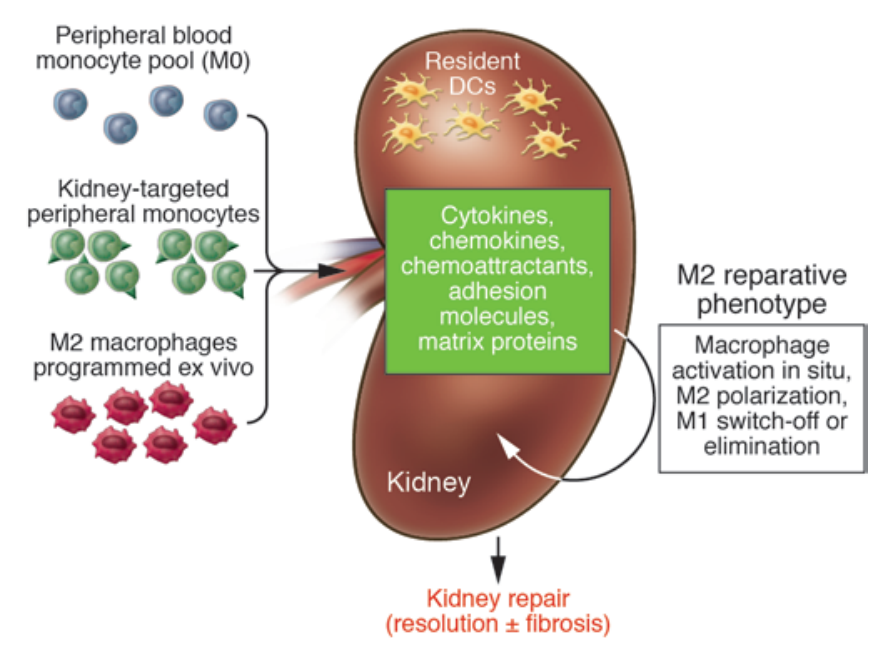

B Secretome-based therapy following kidney injury

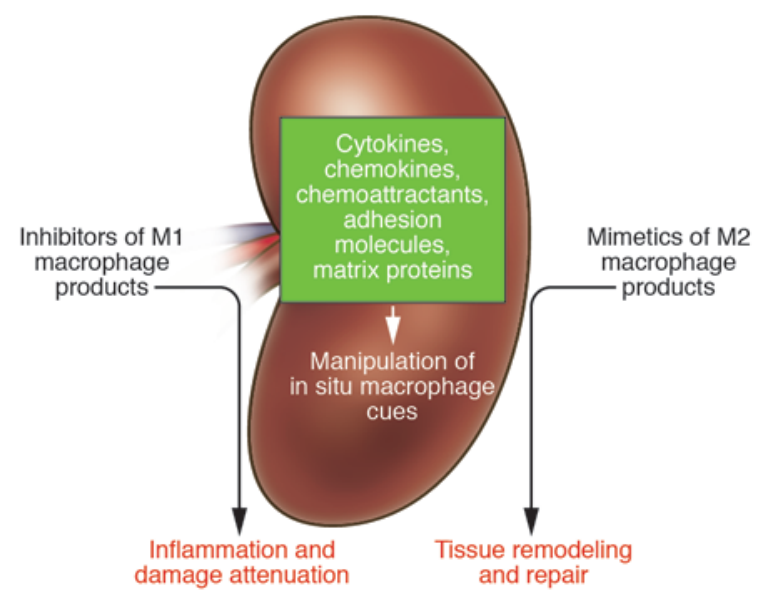

study in mice lacking SHIP, an endogenous inhibitor of the PI3K pathway, suggests that PI3K activation may be necessary for M2 programming and offers another potential priming strategy (51).

\section{Tissue-specific monocyte recruitment}

It is unclear whether chemokines, other chemoattractants, and/or adhesion molecules that attract monocytes to sites of injury also determine monocyte phenotype as they differentiate into macrophages, or whether secondary molecular signals are required to polarize macrophages once they are within a unique microenvironment within a damaged kidney (Figure 2A). Monocyte chemoattractant protein-1 (MCP-1) and its receptor CCR2 are associated with an $\mathrm{M} 1$ response; blocking this pathway experimentally has already been shown to reduce kidney fibrosis (101-103). CCR2 is also a signaling receptor that activates NF-кB. Perhaps this pathway can provide the secondary signal leading to M1 phenotype acquisition. Experimental manipulations that block NF-кB have also been shown to attenuate proteinuric kidney disease (104). However, macrophage phenotype does not always correlate with function. For example, high expression of the fractalkine receptor CX3CR1 aligns with M2 polarity in atherosclerosis and in healing ischemic myocardium and cutaneous wounds, yet CX3CR1 inhibition has been reported to have beneficial effects

\section{Figure 3}

Future therapeutic possibilities for kidney disease. (A) Macrophagebased cellular therapies or therapeutic interventions can be envisaged that capitalize on the specialized macrophage secretome that determines differential function. Monocytes might be manipulated ex vivo to migrate to a damaged kidney, where they are preferentially $\mathrm{M} 2$ polarized, perhaps by inducing receptors to specific chemokines or chemoattractant molecules. Alternatively M2-type macrophages generated ex vivo from peripheral blood monocytes can be administered. Renal dendritic cells and unpolarized macrophages (M0) might also be skewed to an M2 phenotype by therapeutic manipulation of intrarenal molecular signals, such as specific cytokines, chemokines, or ECM proteins, known to direct this process in situ. (B) As more is learned about which soluble secreted macrophage products are associated with renal injury versus repair, single agents or, more likely, a cocktail of biological agents, drugs, and/or small molecules, might be administered to direct tissue recovery. This might include targeting of secondary intracellular signaling cascades that are activated by specific macrophage-derived products.

following renal ischemia (101, 105-107). Clearly more information about the large family of chemokine receptors may offer a unique opportunity to co-manipulate macrophage recruitment and function in pathological states.

\section{Site-specific cues for macrophage polarization}

It is conceivable that critical molecular changes that typify the renal response to injury could be exploited therapeutically. If macrophage polarization occurs locally as a secondary event after recruitment, several innovative therapies can be envisaged that block M1-polarizing pathways. The fact that the currently available TNF- $\alpha$-blocking agents have been disappointing when tested as treatment for aggressive kidney diseases such as Wegener granulomatosis highlights the challenge of translating in vitro observations into complex in vivo milieus (108). This may be a situation wherein a cocktail of agents are needed to effectively "switch off" proinflammatory M1 macrophages.

An alternative approach might be to enhance endogenous pathways that defend against tissue damage by selectively modifying macrophage function. For example, activated HGF has impressive antiinflammatory and antifibrotic effects in several experimental disease models $(109,110)$. Enhanced local expression of HGFactivating proteases, inhibition of endogenous inhibitors of HGF activation (e.g., HGF activator inhibitor type 1[HAI-1] and HAI-2), or administration of exogenous HGF are predicted to attenuate chronic kidney disease, but whether this is accomplished through effects on macrophage programming remains to be determined.

Interventions that re-engineer the molecular composition of wound-associated ECM can be envisaged to skew macrophage polarity. Osteopontin is an ECM protein and monocyte chemoattractant that accumulates in the interstitium in chronic kidney disease (111). An exciting observation made in a skin wounding model was that osteopontin antisense therapy not only reduced inflammation but allowed the skin to heal faster and without scars (112). Deposition of the soluble form of the small proteoglycan biglycan within the ECM can activate a proinflammatory macrophage program via TLRs (113). Renal scar tissue is not simply an inert network of fibrillar collagens, but a dynamic structure comprised of osteopontin, biglycan, and several additional components that may communicate with neighboring cells including macrophages; modification of scar tissue constituents 
offers another therapeutic approach to influencing macrophage phenotype. An area deserving of further investigation is whether resident kidney interstitial macrophages and dendritic cells can be manipulated therapeutically toward a phenotype associated with wound healing (114). Another important question is whether the acquisition of $\mathrm{M} 1$ or $\mathrm{M} 2$ states represents terminal differentiation, or the phenotype is plastic and interconversion is feasible.

\section{Macrophage receptors and soluble secreted products}

The M2 macrophage phenotype is typically associated with highlevel scavenger receptor expression (e.g., the classical SRA/B scavenger receptor MARCO, CD163, the mannose receptor, uPAR, and the AT1; refs. 24, 71, 115). As a group, these receptors serve a multitude of diverse functions, but they share in common the ability to mediate endocytic clearance. This activity is often associated with injury resolution, offering the possibility that macrophage manipulations to augment scavenger receptor levels might be a therapeutic goal.

Additional macrophage profiling studies based on their functional diversity may not only identify new membrane receptors that confer functional specificity but may further elucidate their unique biosynthetic profiles as therapeutic hopefuls (116). For example, it is already known that protection from ROS and NO-derived oxidant species produced by M1 macrophages offers many potential benefits for patients with chronic kidney disease, but development of more potent antioxidant drugs directed against specific targets is desirable. M2-derived products shown to promote tissue repair represent new candidates for therapeutic peptide development. For example, the IL-1 decoy receptor and MMP-13 are M2 products ascribed antiinflammatory and tissue remodeling effects, respectively $(117,118)$. Detailed delineation of the M2 macrophage molecular signature also offers the possibility of new insights into pathways that differentiate scarred from scarless wound healing. Arginase- 1 expression associated with the M2 phenotype converts L-arginine to L-ornithine. While this reaction might be beneficial as it shunts L-arginine away from iNOS and NO-derived species generation, L-ornithine is a precursor for proline, which enhances collagen biosynthesis (119). Identifying an alternative pathway for L-ornithine consumption might reduce scarring in the presence of arginase activity. The IL-13 pathway may offer another therapeutic opportunity. While IL-13 is associated with M2 polarity, the presence of the IL-13 decoy receptor (IL-13R $\alpha_{2}$ ) has been shown to decrease pulmonary fibrosis (120). Systemic Il13 gene therapy reduces renal tubulointerstitial damage and inflammation caused by ischemia-reperfusion in rats (121). More therapeutic targets are likely to emerge as genomics, proteomics, and metabolomics further define the M2 phenotype and function.

\section{Tissue macrophage turnover}

Another aspect of macrophage biology that might be exploited therapeutically pertains to the pathway of elimination. Differentiated macrophages have a finite lifespan and presumably undergo apoptotic death; it seems unlikely that they exit from sites of injury and die elsewhere. Macrophage half-life is limited by the macrophage activation state, the inflammatory milieu, and pro-apoptotic stimuli that determine the frequency of apoptosis $(122,123)$. Differential gene expression studies have identified significant differences in the expression of a subset of apoptosis-related genes (116). Renoprotective interventions might include strategies that increase M2 macrophage survival relative to M1 at sites of kidney injury.

\section{Genotype-specific therapy?}

A major clinical challenge for practicing nephrologists is the lack of precise methods to predict which patients with early chronic kidney disease are at risk for progression to end-stage kidney disease. As the continuum of macrophage functional variation becomes more clearly defined, "staging" studies of injury-associated kidney macrophages present in biopsy specimens may prove to be an informative prognostic indicator. It is also plausible that genetic polymorphisms may determine activity levels for some of these key macrophage functional proteins, as shown for MCP-1, for example, and that sometime in the future peripheral blood genotyping will be useful both to identify patients at risk for progressive kidney disease and to tailor design biological therapeutics $(124,125)$.

In summary, cells of the myeloid lineage are hailed to the kidney in response to injury. In response to local environmental cues, they acquire specialized functions selected from a huge repertoire. Major functional categories include phagocytic scavenging, synthesis of a myriad of soluble secreted products, immune surveillance as APCs, and cellular fusion partners. In a context-dependent manner, functionally polarized intrarenal macrophages may truly serve as "friend" or "foe." As these molecular phenotypes become further defined, new macrophage-based therapies for fibrodestructive disorders such as chronic kidney disease should emerge.

\section{Acknowledgments}

The authors would like to acknowledge research grant support from Kidney Health Australia and the Australian Stem Cell Centre (to S.D. Ricardo) and the NIH (DK54500 and DK44757, to A.A. Eddy).

Address correspondence to: Sharon D. Ricardo, Monash Immunology and Stem Cell Laboratories (MISCL), Monash University, and the Australian Stem Cell Centre, Building 75, STRIP 1, Clayton, Victoria 3800, Australia. Phone: 61-3-9271-1100; Fax: 61-3-92711199; E-mail: sharon.ricardo@med.monash.edu.au.

\footnotetext{
1. Pistole, T.G., and Britko, J.L. 1978. Bactericidal activity of amebocytes from the horseshoe crab, Limulus polyphemus. J. Invertebr. Pathol. 31:376-382.

2. Takahashi, K., and Naito, M. 1993. Development, differentiation, and proliferation of macrophages in the rat yolk sac. Tissue Cell. 25:351-362.

3. Takahashi, K., Yamamura, F., and Naito, M. 1989 Differentiation, maturation, and proliferation of macrophages in the mouse yolk sac: a light-microscopic, enzyme-cytochemical, immunohistochemical, and ultrastructural study. J. Lenkoc. Biol. 45:87-96.

4. Morioka, Y., Naito, M., Sato, T., and Takahashi, K. 1994. Immunophenotypic and ultrastructural heterogeneity of macrophage differentiation in bone marrow and fetal hematopoiesis of mouse in vitro and in vivo. J. Leukoc. Biol. 55:642-651.
}

5. Hume, D.A. 2006. The mononuclear phagocyte system. Curr. Opin. Immunol. 18:49-53.

6. Lichanska, A.M., et al. 1999. Differentiation of the mononuclear phagocyte system during mouse embryogenesis: the role of transcription factor PU.1. Blood. 94:127-138.

7. Shepard, J.L., and Zon, L.I. 2000. Developmental derivation of embryonic and adult macrophages. Curr. Opin. Hematol. 7:3-8.

8. Hume, D.A., et al. 2002. The mononuclear phagocyte system revisited. J. Lenkoc. Biol. 72:621-627.

9. Henson, P.M., and Hume, D.A. 2006. Apoptotic cell removal in development and tissue homeostasis. Trends Immunol. 27:244-250.

10. Rae, F., et al. 2007. Characterisation and trophic functions of murine embryonic macrophages based upon the use of a Csf1r-EGFP transgene reporter. Dev. Biol. 308:232-246.

11. Lichanska, A.M., and Hume, D.A. 2000. Origins and functions of phagocytes in the embryo. Exp. Hematol. 28:601-611.

12. Gordon, S., and Taylor, P.R. 2005. Monocyte and macrophage heterogeneity. Nat. Rev. Immunol. 5:953-964.

13. Rivollier, A., et al. 2004. Immature dendritic cell transdifferentiation into osteoclasts: a novel pathway sustained by the rheumatoid arthritis microenvironment. Blood. 104:4029-4037.

14. Boyle, W.J., Simonet, W.S., and Lacey, D.L. 2003. Osteoclast differentiation and activation. Nature. 423:337-342

15. Geissmann, F., Jung, S., and Littman, D.R. 2003. 
Blood monocytes consist of two principal subsets with distinct migratory properties. Immunity. 19:71-82.

16. Sunderkotter, C., et al. 2004. Subpopulations of mouse blood monocytes differ in maturation stage and inflammatory response. J. Immunol. 172:4410-4417.

17. Fogg, D.K., et al. 2006. A clonogenic bone marrow progenitor specific for macrophages and dendritic cells. Science. 311:83-87.

18. Martinez, F.O., Sica, A., Mantovani, A., and Locati, M. 2008. Macrophage activation and polarization. Front. Biosci. 13:453-461.

19. Mantovani, A., et al. 2004. The chemokine system in diverse forms of macrophage activation and polarization. Trends Immunol. 25:677-686.

20. Chen, Y., and Wood, K.J. 2007. Interleukin-23 and TH17 cells in transplantation immunity: does $23+17$ equal rejection? Transplantation. 84:1071-1074.

21. Ma, H.L., et al. 2008. IL-22 is required for Th17 cellmediated pathology in a mouse model of psoriasislike skin inflammation. J. Clin. Invest. 118:597-607.

22. Krueger, G.G., et al. 2007. A human interleukin$12 / 23$ monoclonal antibody for the treatment of psoriasis. N. Engl. J. Med. 356:580-592.

23. Mantovani, A., Sica, A., and Locati, M. 2007. New vistas on macrophage differentiation and activation. Eur. J. Immunol. 37:14-16.

24. Mantovani, A., Sica, A., and Locati, M. 2005. Macrophage polarization comes of age. Immunity. 23:344-346.

25. Gordon, S. 2003. Alternative activation of macrophages. Nat. Rev. Immunol. 3:23-35.

26. Kluth, D.C. 2007. Pro-resolution properties of macrophages in renal injury. Kidney Int. 72:234-236.

27. Timmer, A.M., and Nizet, V. 2008. IKKbeta/ NF-kappaB and the miscreant macrophage. J. Exp. Med. 205:1255-1259.

28. Wang, Y., et al. 2007. Ex vivo programmed macrophages ameliorate experimental chronic inflammatory renal disease. Kidney Int. 72:290-299.

29. Mosser, D.M. 2003. The many faces of macrophage activation. J. Leukoc. Biol. 73:209-212.

30. Wilson, H.M., Walbaum, D., and Rees, A.J. 2004. Macrophages and the kidney. Curr. Opin. Nephrol. Hypertens. 13:285-290.

31. Bourlier, V., et al. 2008. Remodeling phenotype of human subcutaneous adipose tissue macrophages. Circulation. 117:806-815.

32. Duffield, J.S. 2003. The inflammatory macrophage: a story of Jekyll and Hyde. Clin. Sci. (Lond.) 104:27-38.

33. Mantovani, A., Sozzani, S., Locati, M., Allavena, P., and Sica, A. 2002. Macrophage polarization: tumorassociated macrophages as a paradigm for polarized M2 mononuclear phagocytes. Trends Immunol. 23:549-555.

34. Watkins, S.K., Egilmez, N.K., Suttles, J., and Stout, R.D. 2007. IL-12 rapidly alters the functional profile of tumor-associated and tumor-infiltrating macrophages in vitro and in vivo. J. Immunol. 178:1357-1362.

35. Sica, A., and Bronte, V. 2007. Altered macrophage differentiation and immune dysfunction in tumor development. J. Clin. Invest. 117:1155-1166.

36. Tsuda, Y., et al. 2004. Three different neutrophil subsets exhibited in mice with different susceptibilities to infection by methicillin-resistant Staphylococcus aureus. Immunity. 21:215-226.

37. Jo, S.K., Sung, S.A., Cho, W.Y., Go, K.J., and Kim, H.K. 2006. Macrophages contribute to the initiation of ischaemic acute renal failure in rats. Nephrol. Dial. Transplant. 21:1231-1239.

38. Day, Y.J., Huang, L., Ye, H., Linden, J., and Okusa, M.D. 2005. Renal ischemia-reperfusion injury and adenosine $2 \mathrm{~A}$ receptor-mediated tissue protection: role of macrophages. Am. J. Physiol. Renal Physiol. 288:F722-F731.
39. Ko, G.J., Boo, C.S., Jo, S.K., Cho, W.Y., and Kim, H.K. 2008. Macrophages contribute to the development of renal fibrosis following ischaemia/reperfusion-induced acute kidney injury. Nephrol. Dial. Transplant. 23:842-852.

40. Diamond, J.R., and Pesek-Diamond, I. 1991. Sublethal X-irradiation during acute puromycin nephrosis prevents late renal injury: role of macrophages. Am. J. Physiol. 260:F779-F786.

41. van Goor, H., van der Horst, M.L., Fidler, V., and Grond, J. 1992. Glomerular macrophage modulation affects mesangial expansion in the rat after renal ablation. Lab. Invest. 66:564-571.

42. Duffield, J.S., et al. 2005. Conditional ablation of macrophages halts progression of crescentic glomerulonephritis. Am. J. Pathol. 167:1207-1219.

43. Friedman, S.L. 2005. Mac the knife? Macrophagesthe double-edged sword of hepatic fibrosis. J. Clin. Invest. 115:29-32.

44. Chazaud, B., et al. 2003. Satellite cells attract monocytes and use macrophages as a support to escape apoptosis and enhance muscle growth. J. Cell Biol. 163:1133-1143.

45. Arnold, L., et al. 2007. Inflammatory monocytes recruited after skeletal muscle injury switch into antiinflammatory macrophages to support myogenesis. J. Exp. Med. 204:1057-1069.

46. Pull, S.L., Doherty, J.M., Mills, J.C., Gordon, J.I., and Stappenbeck, T.S. 2005. Activated macrophages are an adaptive element of the colonic epithelial progenitor niche necessary for regenerative responses to injury. Proc. Natl. Acad. Sci. U. S. A. 102:99-104.

47. Ramsay, R.G., et al. 2004. Colony-stimulating factor-1 promotes clonogenic growth of normal murine colonic crypt epithelial cells in vitro. J. Interferon Cytokine Res. 24:416-427.

48. Ide, H., et al. 2002. Expression of colony-stimulating factor 1 receptor during prostate development and prostate cancer progression. Proc. Natl. Acad. Sci. U. S. A. 99:14404-14409.

49. Kacinski, B.M., et al. 1991. FMS (CSF-1 receptor) and CSF-1 transcripts and protein are expressed by human breast carcinomas in vivo and in vitro. Oncogene. 6:941-952.

50. Herbert, D.R., et al. 2004. Alternative macrophage activation is essential for survival during schistosomiasis and downmodulates $T$ helper 1 responses and immunopathology. Immunity. 20:623-635.

51. Rauh, M.J., et al. 2005. SHIP represses the generation of alternatively activated macrophages. Immunity. 23:361-374.

52. Kurts, C., Heymann, F., Lukacs-Kornek, V., Boor, P., and Floege, J. 2007. Role of T cells and dendritic cells in glomerular immunopathology. Semin. Immunopathol. 29:317-335.

53. Chadban, S.J., and Atkins, R.C. 2005. Glomerulonephritis. Lancet. 365:1797-1806.

54. Kalluri, R., Danoff, T.M., Okada, H., and Neilson, E.G. 1997. Susceptibility to anti-glomerular basement membrane disease and Goodpasture syndrome is linked to MHC class II genes and the emergence of $\mathrm{T}$ cell-mediated immunity in mice. J. Clin. Invest. 100:2263-2275.

55. Hume, D.A., Robinson, A.P., MacPherson, G.G., and Gordon, S. 1983. The mononuclear phagocyte system of the mouse defined by immunohistochemical localization of antigen F4/80. Relationship between macrophages, Langerhans cells, reticular cells, and dendritic cells in lymphoid and hematopoietic organs. J. Exp. Med. 158:1522-1536.

56. Brown, F.G., et al. 1999. Up-regulation of macrophage migration inhibitory factor in acute renal allograft rejection in the rat. Clin. Exp. Immunol. 118:329-336.

57. Wyburn, K., et al. 2005. Macrophage-derived interleukin-18 in experimental renal allograft rejection. Nephrol. Dial. Transplant. 20:699-706.

58. Tipping, P.G., and Holdsworth, S.R. 2006. T cells in crescentic glomerulonephritis. J. Am. Soc. Nephrol.
17:1253-1263.

59. Border, W.A., and Noble, N.A. 1994. Transforming growth factor beta in tissue fibrosis. N. Engl.J. Med. 331:1286-1292.

60. Eddy, A.A. 2005. Progression in chronic kidney disease. Adv. Chronic Kidney Dis. 12:353-365.

61. Border, W.A. 1994. Transforming growth factorbeta and the pathogenesis of glomerular diseases. Curr. Opin. Nephrol. Hypertens. 3:54-58.

62. Li, J., et al. 2006. Inhibition of p38 mitogen-activated protein kinase and transforming growth factor-beta1/Smad signaling pathways modulates the development of fibrosis in adriamycin-induced nephropathy. Am. J. Pathol. 169:1527-1540.

63. Iwano, M., et al. 2002. Evidence that fibroblasts derive from epithelium during tissue fibrosis. J. Clin. Invest. 110:341-350.

64. Weitkamp, B., Cullen, P., Plenz, G., Robenek, H., and Rauterberg, J. 1999. Human macrophages synthesize type VIII collagen in vitro and in the atherosclerotic plaque. FASEB J. 13:1445-1457.

65. Tsunawaki, S., Sporn, M., Ding, A., and Nathan, C. 1988. Deactivation of macrophages by transforming growth factor-beta. Nature. 334:260-262.

66. Schnoor, M., et al. 2008. Production of type VI collagen by human macrophages: a new dimension in macrophage functional heterogeneity. J. Immunol. 180:5707-5719.

67. Anders, H.J., et al. 2003. CC chemokine ligand 5/ RANTES chemokine antagonists aggravate glomerulonephritis despite reduction of glomerular leukocyte infiltration. J. Immunol. 170:5658-5666.

68. Yokoo, T., et al. 1999. Prophylaxis of antibodyinduced acute glomerulonephritis with genetically modified bone marrow-derived vehicle cells. Hum. Gene Ther. 10:2673-2678.

69. Yang, J., et al. 2003. Targeting of macrophage activity by adenovirus-mediated intragraft overexpression of TNFRp55-Ig, IL-12p40, and vIL-10 ameliorates adenovirus-mediated chronic graft injury, whereas stimulation of macrophages by overexpression of IFN-gamma accelerates chronic graft injury in a rat renal allograft model. J. Am. Soc. Nephrol. 14:214-225.

70. Nishida, M., et al. 2005. Adoptive transfer of macrophages ameliorates renal fibrosis in mice. Biochem. Biophys. Res. Commun. 332:11-16.

71. Nishida, M., et al. 2002. Absence of angiotensin II type 1 receptor in bone marrow-derived cells is detrimental in the evolution of renal fibrosis. J. Clin. Invest. 110:1859-1868.

72. Zhang, G., et al. 2003. Urokinase receptor deficiency accelerates renal fibrosis in obstructive nephropathy. J. Am. Soc. Nephrol. 14:1254-1271.

73. Wang, Y., et al. 2007. Ex vivo programmed macrophages ameliorate experimental chronic inflammatory renal disease. Kidney Int. 72:290-299.

74. Wilson, H.M., et al. 2005. Inhibition of macrophage nuclear factor-kappaB leads to a dominant antiinflammatory phenotype that attenuates glomerular inflammation in vivo. Am. J. Pathol. 167:27-37.

75. Wilson, H.M., et al. 2002. Bone-marrow-derived macrophages genetically modified to produce IL-10 reduce injury in experimental glomerulonephritis. Mol. Ther. 6:710-717.

76. Humphreys, B.D., and Bonventre, J.V. 2007. The contribution of adult stem cells to renal repair. Nephrol. Ther. 3:3-10.

77. Poulsom, R., et al. 2001. Bone marrow contributes to renal parenchymal turnover and regeneration. J. Pathol. 195:229-235.

78. Rookmaaker, M.B., et al. 2003. Bone-marrowderived cells contribute to glomerular endothelial repair in experimental glomerulonephritis. Am.J. Pathol. 163:553-562.

79. Kale, S., et al. 2003. Bone marrow stem cells contribute to repair of the ischemically injured renal tubule. J. Clin. Invest. 112:42-49.

80. Lin, F., et al. 2003. Hematopoietic stem cells con- 
tribute to the regeneration of renal tubules after renal ischemia-reperfusion injury in mice. J. Am. Soc. Nephrol. 14:1188-1199.

81. Grimm, P.C., et al. 2001. Neointimal and tubulointerstitial infiltration by recipient mesenchymal cells in chronic renal-allograft rejection. N. Engl. J. Med. 345:93-97.

82. Nishida, M., et al. 2003. Renal tubular regeneration by bone marrow-derived cells in a girl after bone marrow transplantation. Am. J. Kidney Dis. 42:E10-E12.

83. Gupta, S., Verfaillie, C., Chmielewski, D., Kim, Y., and Rosenberg, M.E. 2002. A role for extrarenal cells in the regeneration following acute renal failure. Kidney Int. 62:1285-1290.

84. Li, J., Deane, J.A., Campanale, N.V., Bertram, J.F., and Ricardo, S.D. 2006. Blockade of p38 mitogen-activated protein kinase and TGF-beta1/Smad signaling pathways rescues bone marrow-derived peritubular capillary endothelial cells in adriamycin-induced nephrosis. J. Am. Soc. Nephrol. 17:2799-2811.

85. Li, J., Deane, J.A., Campanale, N.V., Bertram, J.F., and Ricardo, S.D. 2007. The contribution of bone marrow-derived cells to the development of renal interstitial fibrosis. Stem Cells. 25:697-706.

86. Masuya, M., et al. 2003. Hematopoietic origin of glomerular mesangial cells. Blood. 101:2215-2218.

87. Camargo, F.D., Chambers, S.M., and Goodell, M.A. 2004. Stem cell plasticity: from transdifferentiation to macrophage fusion. Cell Prolif. 37:55-65.

88. Imasawa, T., et al. 2001. The potential of bone marrow-derived cells to differentiate to glomerular mesangial cells. J. Am. Soc. Nephrol. 12:1401-1409.

89. Ito, T., Suzuki, A., Imai, E., Okabe, M., and Hori, M. 2001. Bone marrow is a reservoir of repopulating mesangial cells during glomerular remodeling. J. Am. Soc. Nephrol. 12:2625-2635.

90. Duffield, J.S., et al. 2005. Restoration of tubular epithelial cells during repair of the postischemic kidney occurs independently of bone marrowderived stem cells. J. Clin. Invest. 115:1743-1755.

91. Lin, F., Moran, A., and Igarashi, P. 2005. Intrarenal cells, not bone marrow-derived cells, are the major source for regeneration in postischemic kidney. J. Clin. Invest. 115:1756-1764.

92. Li, L., Truong, P., Igarashi, P., and Lin, F. 2007. Renal and bone marrow cells fuse after renal ischemic injury. J. Am. Soc. Nephrol. 18:3067-3077.

93. Held, P.K., et al. 2006. In vivo genetic selection of renal proximal tubules. Mol. Ther. 13:49-58.

94. Willenbring, H., et al. 2004. Myelomonocytic cells are sufficient for therapeutic cell fusion in liver. Nat. Med. 10:744-748.

95. Vignery, A. 2000. Osteoclasts and giant cells: macrophage-macrophage fusion mechanism. Int. J. Exp. Pathol. 81:291-304.
96. Bailey, A.S., et al. 2006. Myeloid lineage progenitors give rise to vascular endothelium. Proc. Natl. Acad. Sci. U. S. A. 103:13156-13161.

97. Zhao, Y., Glesne, D., and Huberman, E. 2003. A human peripheral blood monocyte-derived subset acts as pluripotent stem cells. Proc. Natl. Acad. Sci. U. S. A. 100:2426-2431.

98. Havemann, K., Pujol, B.F., and Adamkiewicz, J. 2003. In vitro transformation of monocytes and dendritic cells into endothelial like cells. Adv. Exp. Med. Biol. 522:47-57.

99. Mestas, J., and Hughes, C.C. 2004. Of mice and not men: differences between mouse and human immunology. J. Immunol. 172:2731-2738.

100. Bouhlel, M.A., et al. 2007. PPARgamma activation primes human monocytes into alternative M2 macrophages with anti-inflammatory properties. Cell Metab. 6:137-143.

101. Tacke, F., et al. 2007. Monocyte subsets differentially employ CCR2, CCR5, and CX3CR1 to accumulate within atherosclerotic plaques. J. Clin. Invest. 117:185-194

102.Gordon, S. 2007. Macrophage heterogeneity and tissue lipids. J. Clin. Invest. 117:89-93.

103. Kitagawa, K., et al. 2004. Blockade of CCR2 Ameliorates Progressive Fibrosis in Kidney. Am. J. Pathol. 165:237-246

104. Takase, O., et al. 2003. Gene transfer of truncated IkappaBalpha prevents tubulointerstitial injury. Kidney Int. 63:501-513.

105.Nahrendorf, M., et al. 2007. The healing myocardium sequentially mobilizes two monocyte subsets with divergent and complementary functions. J. Exp. Med. 204:3037-3047.

106.Furuichi, K., Gao, J.L., and Murphy, P.M. 2006. Chemokine receptor CX3CR1 regulates renal interstitial fibrosis after ischemia-reperfusion injury. $\mathrm{Am}$. J. Pathol. 169:372-387.

107. Ishida, Y., Gao, J.L., and Murphy, P.M. 2008. Chemokine receptor CX3CR1 mediates skin wound healing by promoting macrophage and fibroblast accumulation and function. J. Immunol. 180:569-579.

108.Wegener's Granulomatosis Etanercept Trial Research Group. 2005. Etanercept plus standard therapy for Wegener's granulomatosis. N. Engl. J. Med. 352:351-361.

109.Liu, Y. 2004. Hepatocyte growth factor in kidney fibrosis: therapeutic potential and mechanisms of action. Am. J. Physiol. Renal Physiol. 287:F7-F16.

110.Gong, R., Rifai, A., Ge, Y., Chen, S., and Dworkin, L.D. 2008. Hepatocyte growth factor suppresses proinflammatory NFkappaB activation through GSK3beta inactivation in renal tubular epithelial cells. J. Biol. Chem. 283:7401-7410.

111.Ophascharoensuk, V., et al. 1999. Obstructive urop- athy in the mouse: role of osteopontin in interstitial fibrosis and apoptosis. Kidney Int. 56:571-580.

112.Mori, R., Shaw, T.J., and Martin, P. 2008. Molecular mechanisms linking wound inflammation and fibrosis: knockdown of osteopontin leads to rapid repair and reduced scarring. J. Exp. Med. 205:43-51.

113.Schaefer, L., et al. 2005. The matrix component biglycan is proinflammatory and signals through Toll-like receptors 4 and 2 in macrophages. J. Clin. Invest. 115:2223-2233.

114.John, R., and Nelson, P.J. 2007. Dendritic cells in the kidney. J. Am. Soc. Nephrol. 18:2628-2635.

115.Zhang, G., et al. 2003. Urokinase receptor modulates cellular and angiogenic responses in obstructive uropathy. J. Am. Soc. Nephrol. 14:1254-1271.

116. Martinez, F.O., Gordon, S., Locati, M., and Mantovani, A. 2006. Transcriptional profiling of the human monocyte-to-macrophage differentiation and polarization: new molecules and patterns of gene expression. J. Immunol. 177:7303-7311.

117. Vesey, D.A., et al. 2002. Interleukin-1beta induces human proximal tubule cell injury, alpha-smooth muscle actin expression and fibronectin production. Kidney Int. 62:31-40.

118. Fallowfield, J.A., et al. 2007. Scar-associated macrophages are a major source of hepatic matrix metalloproteinase- 13 and facilitate the resolution of murine hepatic fibrosis. J. Immunol. 178:5288-5295.

119.Bronte, V., and Zanovello, P. 2005. Regulation of immune responses by L-arginine metabolism. Nat. Rev. Immunol. 5:641-654.

120.Fichtner-Feigl, S., Strober, W., Kawakami, K., Puri, R.K., and Kitani, A. 2006. IL-13 signaling through the IL-13alpha2 receptor is involved in induction of TGF-beta 1 production and fibrosis. Nat. Med. 12:99-106.

121.Sandovici, M., et al. 2008. Systemic gene therapy with interleukin-13 attenuates renal ischemiareperfusion injury. Kidney Int. 73:1364-1373.

122.Marriott, H.M., et al. 2005. Dynamic changes in Mcl-1 expression regulate macrophage viability or commitment to apoptosis during bacterial clearance. J. Clin. Invest. 115:359-368.

123.Steinberg, B.E., and Grinstein, S. 2008. Pathogen destruction versus intracellular survival: the role of lipids as phagosomal fate determinants. J. Clin. Invest. 118:2002-2011.

124.Flores-Villanueva, P.O., et al. 2005. A functional promoter polymorphism in monocyte chemoattractant protein-1 is associated with increased susceptibility to pulmonary tuberculosis. J. Exp. Med. 202:1649-1658.

125. Kim, H.L., et al. 2002. The polymorphism of monocyte chemoattractant protein- 1 is associated with the renal disease of SLE. Am. J. Kidney Dis. 40:1146-1152. 